\title{
A Retrospective Cohort Study of Nasopharyngeal Carcinoma Screening and Hepatocellular Carcinoma Screening in Zhongshang City
}

\author{
Xia $\mathrm{Yu}^{1}$, Mingfang Ji ${ }^{1}$, Weimin Cheng ${ }^{1}$, Biaohua $\mathrm{Wu}^{1}$, Shifeng Lian ${ }^{1}$, Yun $\mathrm{Du}^{1,2}$, Sumei Cao ${ }^{2}$ \\ 1. Cancer Research Institute of Zhongshan City, Zhongshan City People's Hospital, Zhongshan, Guangdong Province, China; \\ 2. Department of Cancer Prevention Research, Cancer Prevention Center, Sun Yat-sen University Cancer Center, State Key Laboratory of Oncology in \\ Southern China, Collaborative innovation Center for Cancer Medicine, Guangzhou, Guangdong Province, China \\ $凶$ Corresponding author: Mingfang Ji (jmftbh@sina.com) \\ (c) Ivyspring International Publisher. This is an open access article distributed under the terms of the Creative Commons Attribution (CC BY-NC) license \\ (https://creativecommons.org/licenses/by-nc/4.0/). See http://ivyspring.com/terms for full terms and conditions.
}

Received: 2018.06.05; Accepted: 2019.02.24; Published: 2019.04.21

\begin{abstract}
Background: Nasopharyngeal carcinoma (NPC) and hepatocellular carcinoma (HCC) have remained a major burden of public health in Southern China. The screening for early disease in asymptomatic individuals has potentially been the most promising tool to improve cancer treatment outcomes. The present study aims to evaluate the compliance rates and characteristics of cancer incidence in the population of NPC and HCC screening.

Methods: Enzyme-linked immunsorbent assay (ELISA) for Epstein-Barr virus (EBV) antibodies and Hepatitis B surface antigen $(\mathrm{HBsAg})$ was performed in this population. NPC high/medium risk and $\mathrm{HCC}$ high risk individuals were followed-up for a number of years. The compliance rate, cancer incidence and early diagnosis rate of the screened population were statistically analyzed.

Results: (1) In the preliminary screening, the compliance rate for NPC screening was significantly higher than that for HCC screening $(29.3 \%$ vs. $26.2 \% ; P<0.05)$. The compliance rates for screening were positively associated with age in these two screenings $(P<0.01)$. (2) In the NPC screening, the compliance rates for the first year follow-up among NPC high/medium risk individuals were $74.9 \%$, which was higher than that $(60.2 \%)$ for the second year follow-up $(P<0.05)$. The compliance rates for fiberoptic endoscopy among high risk individuals decreased along with the frequency of screening $(P<0.016)$. The rates of missed diagnosis by non-compliance and the poor diagnostic accuracy of indicators were 3.3\% and 3.3\%, respectively. The average annual incidence and early diagnosis rate of the compliers were higher than those of the non-compliers $(94.3$ per 100,000 vs. 29.0 per 100,$000 ; P<0.05$ and $77.8 \%$ vs. $18.5 \%$; $P<0.05$ ). (3) In the preliminary HCC screening, the compliance rate for ultrasonography among high risk individuals was $61.8 \%$. The compliance rates for the follow-up were unsatisfactory. The rates of missed diagnosis by non-compliance and the poor diagnostic accuracy of indicators were $12.3 \%$ and $24.6 \%$, respectively. There was no significant differences in average annual incidence and the rate of early diagnosis between compliers and non-compliers $(79.4$ per 100,000 vs. 54.6 per $100,000, P>0.05 ; 49.1 \%$ vs. $38.5 \%, P>0.05)$.

Conclusion: The compliance rates for NPC and HCC screening needs to be improved. In particular, public health policies for HCC should be implemented. The present NPC screening could be the preferred strategy. However, the efficiency of HCC screening remains substantially unsatisfactory and needs to be further discussed.
\end{abstract}

Key words: nasopharyngeal carcinoma, hepatocellular carcinoma, screening, compliance

\section{Introduction}

Nasopharyngeal carcinoma (NPC) and hepatocellular carcinoma (HCC) are common malignant tumors in China that severely threaten health $[1,2]$. Zhongshan City in Southern China is one of the high-incidence areas of NPC and HCC [3]. Since the 1980s, the Research Institute of Zhongshan City has launched several pilot efforts to conduct cancer screenings. In the present study, a total of 40,750 and 
17,082 individuals were enrolled for NPC screening and HCC screening, respectively. Previous studies have rarely analyzed the situation of compliance in cancer screening. The first purpose of the present study was to investigate the compliance rates in preliminary screenings and follow-ups. The second purpose of the present study was to evaluate the average annual incidence and early diagnosis rate between compliers and non-compliers in NPC and HCC screening.

\section{Materials and Methods}

\section{Study population, serologic test and screening protocol}

\section{NPC screening}

NPC screening was conducted in Xiaolan, Ming Zhong and Gang Kuo Town, which are communities in southern China. A total of 40,750 individuals, who were 30-69 years old, were recruited from 2009 to 2014. Two screening markers, VCA/IgA (Euroimmun, Lubeck, Germany) and EBNA1/IgA (Zhongshan Bio-tech, Zhongshan, China) were tested using the ELISA method. The levels of these seromarkers were assessed by photometric measurement, according to manufacturers' instructions, and were standardized by calculating the ratio of the optical density (OD) of the sample over that of the reference control (rOD) [4]. The combination of these two seromarkers, with a sensitivity of $92.8 \%$, a specificity of $91.6 \%$ and an ROC of 0.97 , was identified, and a prediction formula was developed: $\operatorname{Logit} P R O B=-3.934+2.203 \times \mathrm{VCA} / \mathrm{IgA}+$ $4.797 \times$ EBNA1/IgA [5]. On the basis of a predefined serologic algorithm, these participants were classified into three subgroups: high-risk (PROB $\geq 0.98$ ), medium-risk (0.98>PROB $\geq 0.65)$, and low-risk $(\mathrm{PROB}<$ 0.65). Except for patients diagnosed with NPC, the remaining high/medium risk individuals were followed up annually with blood samples, which were examined for EBV-related antibodies. Fiberoptic endoscopy was performed by local otorhinolaryngologists at Zhongshan People's Hospital when the serologic result reached the definition of high-risk. Nasopharyngeal biopises also were performed when suspicious lesions were observed during the endoscopy procedure. NPC cases were identified by the research team among the high risk group, and as a complement, these were identified through linkage to cancer registries annually. NPC diagnoses from the low risk group were mainly ascertained through cancer registries. NPC was classified according to the World Health Organization classification [6], and tumor stage was defined according to the 2008 staging system of China [7]. Stages I and II were the early stages. The present survey was carried out for up for one screening cycle.

\section{HCC screening}

HCC screening was conducted in Xiaolan Town. A total of 17,966 individuals, who were within 35-64 years old for males and within 45-64 years old for females, were recruited in 2012. HBsAg was tested using the ELISA method. The level of HBsAg was assessed by photometric measurement, according to manufacturer's instructions, and standardized by calculating the ratio of the OD of the sample over that of the rOD. If a specific rOD was greater than 1, the sample was regarded as positive. The biannual screening of serum AFP level and ultrasonography was performed in HBsAg-positive individuals (high risk individuals of HCC). Tumor stage was defined according to the Barcelona Clinic Liver Cancer (BCLC) classification staging system [8]. BCLC-0/A was the early stage, while BCLC-B was the intermediate stage. The survey was carried out up to December 2015.

All serum samples for NPC and HCC screening collected during enrollment and on subsequent occasions were tested in separate batches. These were maintained at $4^{\circ} \mathrm{C}$ and used within one month, or kept at $-80^{\circ} \mathrm{C}$ for longer periods.

\section{Statistical analysis}

The rates of early diagnosis and the average incidence for NPC and HCC were compared using chi-square test. The correlation between groups was analyzed by correlation analysis of multi-class test. All statistical analyses were two-sided. A $P$-value $<0.05$ were considered statistically significant. All analyses were performed using the SPSS 20.0 software.

\section{Results}

\section{Characteristics of gender and age for subjects in the preliminary NPC and HCC screening}

The target population for the NPC and HCC screening were 138,891 and 68,551 , respectively. In fact, the population recruited during the preliminary screening was 40,750 and 17,966 , respectively. The compliance rate among the target population in the NPC screening was $29.3 \%$, which was higher than that $(26.2 \%)$ in the HCC screening $(P<0.01)$. In this population, females had a higher compliance rate than that males in the NPC screening $(P<0.01)$. However, in the HCC screening, males had a higher compliance rate than females $(P<0.01)$.

There were significant differences in the compliance rate for these two screenings for the different age brackets $(P<0.01)$. The compliance rates for the target population were positively associated 
with age $(P<0.01)$. The correlation coefficient was 0.173 and 0.136 for NPC and HCC screening, respectively. The compliance rates were low within the age groups of 30-39 and 65-69 years old, increased rapidly within the age group of 40-44 years old, and peaked within the age group of 50-59 years old for NPC screenings and 50-64 years old for HCC screening, respectively (Table 1 ).

\section{Follow-up survey for the NPC and HCC screening}

The follow-up survey among high/medium risk individuals in the NPC screening

Within the first turn of NPC screening, a total of 3,145 subjects were classified as high/medium risk individuals. The compliance rate among high/ medium risk individuals was $76.3 \%(2,356 / 3,087)$ in the first year of follow-up, and this became higher than $60.3 \%(1,847 / 3,063)$ in the second year of follow-up $(P<0.01)$.

Follow-up survey for fiberoptic endoscopy among high risk individuals in the NPC screening

Within the first turn of NPC screening, a total of 895 subjects were classified as high risk individuals. In one screening cycle, there were significant differences in compliance rate among NPC high risk individuals who needed to undergo fiberoptic endoscopy $(P<$ 0.01). The compliance rates for fiberoptic endoscopy among high risk individuals during the preliminary screening and at two years of follow-up were $80.3 \%$ (719/895), 72.3\% (566/783), and 64.5\% (309/475), respectively. However, these compliance rates decreased along with the frequency of screening $(P<0.0167$, Table 2).

Follow-up survey for AFP and ultrasonography among high risk individuals in the $\mathrm{HCC}$ screening

Within the first turn of HCC screening, 2,848 of
17,966 subjects were classified as high risk individuals. All of these subjects were tested for serum AFP levels. However, merely 1,760 high risk individuals were examined for ultrasonography. The compliance rate for ultrasonography was $61.8 \%$. During the screening period, five follow-ups were conducted. The compliance rates for these follow-ups among high risk individuals were $40.0 \%(1,134 / 2,835), 56.8 \%$ $(1,599 / 2,835), \quad 47.5 \% \quad(1,324 / 2,813), \quad 64.9 \% \quad(1,782 /$ $2,790)$, and $54.2 \%(1,487 / 2,745)$, respectively. For high risk individuals, $80.3 \%(2,286)$ were tested for AFP at least once, $68.4 \%(1,948)$ were tested for AFP at least twice, $75.5 \%(2,151)$ were examined for ultrasonography at least once, and $63.5 \%(2,848)$ were examined for ultrasonography at least twice during the follow-up period.

\section{The rates of missed diagnosis in the NPC and HCC screening}

Within the first turn of NPC screening, 58 NPC patients were detected. During the follow-up, a total of 32 NPCs were diagnosed. Among them, 26 NPCs were identified during the routine follow-up, three NPCs were high risk individuals who exited the follow-up period, and three NPCs were detected from low risk individuals. The rates of missed diagnosis by non-compliance and the poor diagnostic accuracy of indicators were $3.3 \%$ and $3.3 \%$, respectively.

Within the first turn of HCC screening, 20 HCC patients were detected. During the follow-up, a total of 37 NPCs were diagnosed. Among them, 16 HCCs were identified during the routine follow-up, seven HCCs were high risk individuals who exited the follow-up period, and 16 HCCs were detected from HBsAg-negative individuals. The rates of missed diagnosis by non-compliance and the poor diagnostic accuracy of indicators were $12.3 \%$ and $24.6 \%$, respectively.

Table 1. Characteristics of gender and age for compliers in the NPC and HCC screening

\begin{tabular}{|c|c|c|c|c|c|c|}
\hline \multirow[t]{2}{*}{ Compliers } & \multicolumn{3}{|l|}{ NPC screening } & \multicolumn{3}{|l|}{ HCC screening } \\
\hline & Total number & Compliance rate & Statistic values & Total number & Compliance rate & Statistic values \\
\hline Number & 40,750 & 29.3 & & 17,966 & 26.2 & \\
\hline \multicolumn{7}{|l|}{ Gender } \\
\hline Male & 16,631 & 25.2 & $\chi^{2}=1039.37, P<0.01$ & 10,555 & 32.8 & $x^{2}=1363.89, P<0.01$ \\
\hline Female & 24,119 & 33.1 & & 7,411 & 20.4 & \\
\hline \multicolumn{7}{|l|}{ Age(year) } \\
\hline $30 \sim$ & 4,248 & 17.5 & $\chi^{2}=4308.52, P<0.01$ & - & - & $X^{2}=1289.73, P<0.01$ \\
\hline $35 \sim$ & 6,019 & 22.2 & & 1,820 & 15.9 & \\
\hline $40 \sim$ & 7,500 & 34.3 & & 2,209 & 22.4 & \\
\hline $45 \sim$ & 7,120 & 31.4 & & 4,280 & 24.9 & \\
\hline $50 \sim$ & 6,866 & 35.4 & & 3,073 & 30.6 & \\
\hline $55 \sim$ & 6,469 & 42.8 & & 3,679 & 33.4 & \\
\hline $60 \sim$ & 2,068 & 31.3 & & 2,905 & 32.4 & \\
\hline $65 \sim 69$ & 460 & 24.9 & & - & - & \\
\hline
\end{tabular}

Note: “-" represents that the ages are not target populations for screening 


\section{Analysis of the diagnosis of patients in compliers and non-compliers in the NPC and HCC screening}

The average annual incidences of the non-compliers, who were within 30-69 years old in the NPC screening, and were within 35-64 years old for males and 45-64 years old for female in the HCC screening, were $29.0 / 10^{5}$ and $54.6 / 10^{5}$, respectively, according to the registered data of the Cancer Institute of Zhongshan City. The average annual incidence of compliers in the NPC screening $\left(94.3 / 10^{5}\right)$ was higher than that of non-compliers $(P<0.05)$. However, there was no significant difference in average annual incidence between compliers $\left(79.4 / 10^{5}\right)$ and non-compliers in the HCC screening $(P>0.05)$.

The early diagnosis rates of compliers were $77.8 \%(70 / 90)$ and $49.1 \%(28 / 57)$ in the NPC and HCC screening, respectively. The early diagnosis rate of compliers in NPC screening was higher than that of non-compliers $(18.5 \%, 5 / 27 ; P<0.05)$. However, there was no significant difference in early diagnosis rate between compliers and non-compliers in the HCC screening (38.5\%, 40/104; $P>0.05$; Table 2 ).

Table 2. Comparison of average annual incidences and rates of early diagnosis in the NPC and HCC screening

\begin{tabular}{lllll}
\hline Group & \multicolumn{2}{l}{$\begin{array}{l}\text { Average annual } \\
\text { incidence/100,000 person-year }\end{array}$} & \multicolumn{2}{l}{ Rate of early diagnosis } \\
\cline { 2 - 5 } & $\begin{array}{l}\text { NPC } \\
\text { screening } \\
\text { cohort }\end{array}$ & $\begin{array}{l}\text { HCC } \\
\text { screening } \\
\text { cohort }\end{array}$ & $\begin{array}{l}\text { NPC } \\
\text { screening } \\
\text { cohort }\end{array}$ & $\begin{array}{l}\text { HCC } \\
\text { screening } \\
\text { cohort }\end{array}$ \\
\hline Compliers & 94.3 & 79.4 & $77.8 \%$ & $49.1 \%$ \\
Non-compliers & 29.0 & 54.6 & $18.5 \%$ & $38.5 \%$ \\
Statistic value & $\chi^{2}=106.6$, & $\chi^{2}=0.03$, & $\chi^{2}=31.7$, & $\chi^{2}=1.7$, \\
& $P=0.001$ & $P=0.868$ & $P=0.000$ & $P=0.19$ \\
\hline
\end{tabular}

\section{Discussion}

NPC and HCC ranked as the third and second highest cancer incidence in Guangdong Province in 2012, respectively [9]. NPC and HCC tend to present at an advanced stage at diagnosis, and have a relatively poor survival rate after diagnosis. Previous studies have shown that screening for NPC and HCC can significantly reduce the mortality rate associated with this disease, and increase overall survival $[10,11]$. The Research Institute of Zhongshan City launched the new NPC screening and HCC screening since 2009, according to the early diagnosis and early treatment protocol of the Ministry of Health in China [12]. The aim of the present study was to evaluate the compliance rates and characteristics of cancer incidences for compliers on the basis of data obtained from large-scale samples and long-term follow-ups during the screening.

A meta-analysis [13] revealed that the compliance rate in colorectal cancer screening was $33.3 \%$, which was lower in developing countries [14]. In the present study, the compliance rate among the target population in the NPC screening was $29.3 \%$, which was higher than that the rate in the HCC screening $(26.2 \%)$. It is possible that this was closely correlated to the public health education in NPC screening during the period of the Eleventh National Science and Technology Support Program of China. In the NPC preliminary screening, the compliance rate for females was higher than that of males. This was consistent with an analysis that the awareness of NPC risk factors in females was stronger than that of males [15]. In the HCC preliminary screening, the compliance rate for males was higher than that of females. This corresponded to the percentage of individuals with HBsAg-positive and the awareness of HBV status [16]. The compliance rates among target population were positively associated with age in these two screenings, and was in line with the NPC and HCC incidence trends. The compliance rates were low within the age range of 30-39 and 65-69 years old. Thus, health education for cancer should be especially implemented among different populations. A previous study [15] reported that the main reasons for populations that did not attend the NPC screening were the psychological burden after diagnosis and ignorance of NPC screening. Another survey [17] revealed that more than half of non-compliers had psychological burden of HCC. This was associated with the poor prognosis and low overall survival of HCC $[18,19]$. Hence, this shows that the awareness of the benefits to survival of the NPC and HCC screening should be increased. A community-based participatory research (CBPR) would be beneficial to increase the compliance rate for cancer screening [20]. However, it remains unclear whether CBPR is suitable for public health in China.

One of the principles for cancer screening is follow-up. Low compliance rate was the main factor that influenced screening efficiency. In the NPC screening, the compliance rates for follow-up among NPC high/medium risk individuals and for fiberoptic endoscopy among high risk individuals decreased along with the frequency of screening.

The rates of missed diagnosis by non-compliance and the poor diagnostic accuracy of indicators were $3.3 \%$ and $3.3 \%$, respectively. The average annual incidence and early diagnosis rate in compliers were higher than that of non-compliers. Screening has been shown to effectively diagnose early-stage NPC. The new NPC screening could be the preferred serodiagnositc strategy for long-term NPC screening [21]. The overall survival for NPC has increased in recent years [22]. However, prospective randomized 
trials are needed to provide sound evidence for the potential effect of screening in reducing NPC-specific mortality [23].

In the HCC screening, the compliance rate for ultrasonography among high risk individuals in the preliminary screening was $61.8 \%$. For the five continuous follow-ups, the compliance rates were $40.0 \%, 56.8 \%, 47.5 \%, 64.9 \%$ and $54.2 \%$, respectively. The lower rates were associated with the shorter screening interval. The rates of missed diagnosis by non-compliance and the poor diagnostic accuracy of indicators were $12.3 \%$ and $24.6 \%$, respectively. There was no significant difference in average annual incidence and early diagnosis rate between compliers and non-compliers. The early diagnosis rate in the HCC screening was significantly lower than that in the CHC-2000 trial [24]. The present screening programme (HBsAg+AFP+ultrasonography) may miss a part of HCC, which includes HBsAg-negative, $\mathrm{HCV}$ and non-viral infections. Cohort studies have revealed that increasing the compliance rate and early diagnosis rate of HCC could increase life expectancy and reduce HCV-related HCC mortality [25]. Thus, future work is warranted to develop more reasonable screening programs, better training for ultrasonography operators, improve the education of patients in terms of compliance, and better quality follow-up to enhance HCC screening efficiency. A recent study [26] demonstrated that the combined decteion of $\mathrm{HBsAg}$, anti-HCV and AFP as a preliminary screening indicator could improve the positive predictive value of HCC high risk individuals. Other laboratory researches $[27,28]$ have reported that the cfDNA methylation analysis and serum metabolite biomarker panel exhibited good diagnostic performance for the early detection of HCC. Therefore, it could be considered that these discoveries have potential in the clinical practice of the HCC diagnosis and screening.

\section{Acknowledgements}

This study was supported by grants from the National Science Foundation of China (NO. 81572062 and NO. 81672098), the 20 th National Science and Technology Support Program of China (2014BAI09 B10), the Sun Yat-sen University Clinical Research 5010 Program (2013012), and the Early Detection of Cancer Project in China (2010-13).

\section{Competing Interests}

The authors have declared that no competing interest exists.

\section{References}

1. Wei KR, Zheng RS, Zhang SW, et al. Nasopharyngeal carcinoma incidence and mortality in China, 2013. Chin J Cancer. 2017;36(1):90.
2. Chen $\mathrm{W}$, Zheng $\mathrm{R}$, Zhang $\mathrm{S}$, et al. Cancer incidence and mortality in China in 2013: an analysis based on urbanization level. Chin J Cancer Res. 2017;29(1):1-10.

3. Zhiheng Liang, Huishan Cen, Kuangrong Wei. An analysis of cancer incidence in Zhongshan, 2011 [article in Chinese]. China Cancer. 2015;24(8):645-648

4. Ji MF, Huang QH, Yu X, et al. Evaluation of plasma Epstein-Barr virus DNA load to distinguish nasopharyngeal carcinoma patients from healthy high-risk population in Southern China. Cancer. 2014;120(9) :1353-1360.

5. Liu Y, Huang Q, Liu W, Liu Q, et al. Establishment of VCA and EBNA1 IgA-based combination by enzyme-linked immunosorbent assay as preferred screening method for nasopharyngeal carcinoma: a two-stage design with a preliminary performance study and a mass screening in southern China. Int J Cancer. 2012;131(2):406-416.

6. Shanmugaratnam K, Sobin LH. The World Health Organization histological classification of tumours of the upper respiratory tract and ear.A commentary on the second edition. Cancer. 1993;71(8):2689-2697.

7. Lin ZX, Yang ZN, Zhan YZ, et al. Application study of the 2008 staging system of nasopharyngeal carcinoma [article in Chinese]. Ai Zheng. 2009;28(10):1029-1032.

8. Llovet JM, Bru C, Bruix J. Prognosis of hepatocellular carcinoma: the BCLC staging classification. Semin Liver Dis. 1999;19(3):329-338.

9. Ruilin Meng, Yujun $\mathrm{Xu}$, Linfeng Lin, et al. Cancer incidence and mortality in Guangdong Province, 2012 [article in Chinese]. China Cancer. 2016;25(12):933-941.

10. Qing Liu, Jin-Ou Chen, Qi-Hong Huang, et al. Trends in the survival of patients with nasopharyngeal carcinoma between 1976 and 2005 in Sihui, China: a population-based study. Chin J Cancer. 2013;32(6):325-333.

11. Yeh YP, Hu TH, Cho PY, et al. Evaluation of abdominal ultrasonography mass screening for hepatocellular carcinoma in Taiwan. Hepatology. 2014;59(5):1840-1849.

12. Expert Committee on Cancer Early Diagnosis and Early Treatment. Protocol of the early diagnosis and early treatment for cancer(Edition 2011) [article in Chinese]. Beijing: The People's Health Publishing House. 2011;110-171.

13. Hassan C, Giorgi Rossi P, Camilloni L, et al. Meta-analysis: adherence to colorectal cancer screening and the detection rate for advanced neoplasia,according to the type of screening test. Aliment Pharmacol Ther. 2012;36(10):929-940.

14. Gakidou E, Nordhagen S, Obermeyer Z. Coverage of cervical cancer screening in 57 countries. PLoS Med. 2008;5(6):132.

15. Xinghua Li, Mingfang Ji, Biaohua Wu, et al. Analysis of questionnaire investigation on knowledge about prevention and treatment of nasopharyngeal carcinoma among 1,453 residents in Xiaolan Town, Zhongshan City, Guangdong Province [article in Chinese]. Chin J Cancer Prev Treat. 2011;18(9): 647-650.

16. Xia Yu, Mingfang Ji, Weiming Cheng, et al. Situation and problem on screening of liver cancer in high-incedence area of Xiaolan, Zhongshan City [article in Chinese]. Cancer Res Prev Treat. 2016;43(4): 287-290.

17. Li Deng, Mingfang Ji, Qianjin Feng, et al. An analysis of questionnaire survery of awareness of liver cancer prevention and control among 1,685 HBV carriers [article in Chinese]. China Cancer. 2017;26(4):249-252.

18. Rongshou Zheng, Tingting Zuo, Hongmei Zeng, et al. Mortality and survival analysis of liver cancer in China [article in Chinese]. Chin J Oncol. 2015;37(9): 697-702.

19. Xiao L, Zhang RL, Zhang $\mathrm{H}$, et al. Comparison of the clinical characteristics and survival between Uyghur patients with hepatitis virus-related and non-B, non-C hepatocellular carcinoma in Xinjiang, China. Chin J Cancer Res. 2015;27(3):279-287.

20. Dias S, Gama A. Community-based participatory research in public health: potentials and challenges. Rev Panam Salud Publica. 2014;35(2):150-154.

21. Xia Yu, Mingfang Ji, Weimin Cheng, et al. Assessment of the long-term diagnostic performance of the new serological screening in large-scale nasopharyngeal carcinoma screening. Journal of Cancer. 2018;9(12):2093-2097.

22. Coghil AEl, Hsu WL, Pfeiffer RM, et al. Epstein-Barr virus serology as a potential screening marker for nasopharyngeal carcinoma among high-risk individuals from multiplex families in Taiwan. Cancer Epidemiol Biomarkers Prev. 2014;23(7):1213-1219.

23. Pepe MS, Etzioni R, Feng Z, et al. Phases of biomarker development for early detection of cancer. J Natl Cancer Inst. 2001;93(14):1054-1061.

24. Trinchet JC, Chaffaut C, Bourcier V, et al. Ultrasonographic surveillance of hepatocellular carcinoma in cirrhosis: a randomized trial comparing 3and 6-month periodicities. Hepatology. 2011;54(6):1987-1997.

25. Abbas Mourad, Sylvie Deuffic-Burban, Nathalie Ganne-Carrie, et al. Hepatocellular carcinoma screening in patients with compensated hepatitis C virus (HCV)-related cirrhosis aware of their HCV status 
improves survival: a modeling approach. Hepatology. 2014;59(4):1471-1481.

26. Sun Hao, Ji Mingfang, Zhang Changhua, et al. Feasibility study of combined detection of multiple indicators in hepatocellular carcinoma screening [article in Chinese]. Chinese Archives of General Surgery. 2016;10(4): 294-297.

27. Rui-hua Xu, Wei Wei, Michal Krawczyk, et al. Circulating tumour DNA methylation markers for diagnosis and prognosis of hepatocellular carcinoma. Nat Mater. 2017;16(11):1155-1161.

28. Luo P, Yin P, Hua R, et al. A Large-scale, multi-center serum metabolite biomarkers identification study for the early detection of hepatocellular carcinoma. Hepatology. 2017 Sep 28.doi:10.1002/hep.29561.[Epub ahead of print]. 